\title{
Identifying the neural substrates of intrinsic motivation during task performance
}

\author{
Woogul Lee ${ }^{1}$ Johnmarshall Reeve ${ }^{2}$
}

Published online: 21 June 2017

(C) Psychonomic Society, Inc. 2017

\begin{abstract}
Intrinsic motivation is the inherent tendency to seek out novelty and challenge, to explore and investigate, and to stretch and extend one's capacities. When people imagine performing intrinsically motivating tasks, they show heightened anterior insular cortex (AIC) activity. To fully explain the neural system of intrinsic motivation, however, requires assessing neural activity while people actually perform intrinsically motivating tasks (i.e., while answering curiosity-inducing questions or solving competence-enabling anagrams). Using event-related functional magnetic resonance imaging, we found that the neural system of intrinsic motivation involves not only AIC activity, but also striatum activity and, further, AIC-striatum functional interactions. These findings suggest that subjective feelings of intrinsic satisfaction (associated with AIC activations), reward processing (associated with striatum activations), and their interactions underlie the actual experience of intrinsic motivation. These neural findings are consistent with the conceptualization of intrinsic motivation as the pursuit and satisfaction of subjective feelings (interest and enjoyment) as intrinsic rewards.
\end{abstract}

Keywords Intrinsic motivation $\cdot$ Anterior insular cortex (AIC) $\cdot$ Striatum $\cdot$ Functional connectivity $\cdot$ Functional magnetic resonance imaging ( $\mathrm{MMRI}$ )

Woogul Lee

woogul@knue.ac.kr

Johnmarshall Reeve

reeve@korea.ac.kr

1 Department of Education, Korea National University of Education, Cheongju-si, Chungbuk 28173, South Korea

2 Department of Education, Brain and Motivation Research Institute, Korea University, Seoul 02841, South Korea
Intrinsic motivation is the inherent tendency to seek out novelty and challenge, to explore and investigate, and to stretch and extend one's capacities (Ryan \& Deci, 2000, 2017). It is a naturally occurring inclination toward exploration, spontaneous interest, and environmental mastery that emerges when the individual anticipates discovering new information (exploration), learning something new (spontaneous interest), and developing and extending existing capacities (environmental mastery). These volitional pursuits generate subjective feelings of interest and enjoyment as the individual gains new information (curiosity satisfaction) and develops greater capacity (competence satisfaction). These subjective feelings (interest and enjoyment) signal experiences of intrinsic satisfaction from a job well done that then function as intrinsic rewards to encourage volitional present and future engagement in that task, activity, or environment (Deci, 1992; Krapp, 2005). Thus, intrinsic motivation is the desire to seek out novelty and explore (e.g., curiosity) and to seek out and master optimal challenge (e.g., competence) for no reason other than the resulting feelings of interest and enjoyment (Abuhamdeh, Csikszentmihalyi, \& Jalal, 2015; Deci \& Ryan, 1985; Loewenstein, 1994; Reeve, 1989).

Many psychologists have shown the theoretical and practical importance of intrinsic motivation. When people engage in a task out of high (vs. low) intrinsic motivation, they show greater exploration (Reeve \& Nix, 1997), effort (Jang, Kim, \& Reeve, 2016), persistence (Vansteenkiste \& Deci, 2003), learning (Vansteenkiste, Simons, Lens, Sheldon, \& Deci, 2004), and performance (Cerasoli, Nicklin, \& Ford, 2014). Furthermore, intrinsic motivation generates a higher quality of engagement, learning, and performance than is observed with extrinsic motivation (i.e., the motivation or reason to act that arises from the offering of attractive environmental incentives and consequences that are separable from the 
activity itself, such as the offering of money, points, or a food reward; Deci, Koestner, \& Ryan, 1999; Ryan \& Deci, 2017).

Although neuroscientific studies on extrinsic motivation are abundant, neuroscientific studies of intrinsic motivation are not (Reeve \& Lee, 2012). Nevertheless, some neuroscientific studies have started to examine intrinsic motivation and intrinsic motivational processes (e.g., curiosity and competence). For example, both Kang and her colleagues (2009) and Gruber, Gelman, and Ranganath (2014) examined the neural basis of intrinsic motivation when participants answered curiosity-provoking trivia questions. Similarly, Murayama, Matsumoto, Izuma, and Matsumoto (2010) examined the neural bases of intrinsic motivation when participants engaged in a "moderately challenging and inherently interesting" stopwatch task. In these studies, striatum activity was repeatedly observed when participants were intrinsically motivated during the task performance phase. Striatum activity is known to be associated with reward processing - which includes integrating reward-related information and generating reward-based approach behavior - and has been observed to play a crucial role in extrinsic motivation (Delgado, 2007; Haber \& Knutson, 2010; O'Doherty, 2004). These results suggest the functional importance of reward processing not only in extrinsic motivation, but also in curiosity-based and competence-based intrinsic motivation.

In a parallel program of research, other researchers have sought to identify the unique neural basis of intrinsic as compared to extrinsic motivation (Lee \& Reeve, 2013; Lee, Reeve, Xue, \& Xiong, 2012). Specifically, these researchers examined neural activity while participants imagined an intrinsically motivating activity (e.g., writing an enjoyable paper) and compared it to neural activity while participants imagined the same activity but with extrinsic motivation (e.g., writing an extra-credit paper). The results showed that the anterior insular cortex (AIC) was uniquely activated during intrinsically rather than during extrinsically motivating situations. Considering that AIC activity is associated with the processing of bodily satisfaction (Goldstein et al., 2009; Naqvi \& Bechara, 2009) and subjective feelings (Craig, 2009; Damasio, 1999), the positive subjective feelings that arise spontaneously from activity-generated feelings of satisfaction seem to be an important source of intrinsic motivation. This idea has been supported by findings showing rather large and positive correlations between AIC activity during intrinsically motivating situations and participants' self-reported general daily intrinsic satisfaction (i.e., feeling competent and autonomous; Lee \& Reeve, 2013).

Although the previous studies identified AIC activity as a unique neural basis of intrinsic motivation, neural activity related to reward processing (e.g., striatum activity) was not observed in these studies (Lee \& Reeve, 2013; Lee et al., 2012). Two possible reasons for this omission seem likely. First, because neural differences between intrinsic and extrinsic motivation were examined in these studies, common neural activity for both types of motivation (e.g., striatum activity) could not be observed, given the experimental design. That is, these studies compared the neural bases of intrinsic versus extrinsic motivation, rather than the neural bases of high versus low intrinsic motivation (i.e., the presence vs. absence of intrinsic motivation). Therefore, in the present study we contrasted the neural activity when people performed intrinsically motivating versus comparable, but not intrinsically motivating, tasks (i.e., high vs. low intrinsic motivation). Second, the previous studies examined neural activity while people were only imagining engaging in activities. Even though actual behavior and imagined behavior both tend to activate common neural systems (Decety, 1996; Jeannerod, 2001), in the case of intrinsic motivation there could be some neural differences between actual experiences versus imagined feelings. Therefore, it was necessary to examine neural activity during actual task performance.

To create conditions under which participants would experience high versus low intrinsic motivation, we used two tasks - the first of which contrasted curiosity-inducing (intrinsically motivating) versus non-curiosity-inducing question answering (Kang et al., 2009; Loewenstein, 1994), and the second of which contrasted competence-enabling (intrinsically motivating) versus non-competence-enabling anagram solving (Reeve, 1989; Ryan \& Deci, 2000). Because intrinsic motivation emerges out of satisfaction related to both curiosity and competence, we expected participants' neural activity and self-reported task interest to be essentially the same on both tasks (i.e., two different operational definitions of an intrinsically motivating task).

We hypothesized that AIC activity would be related to the experimentally manipulated high versus low in-vivo actual experience of intrinsic motivation (H1). H1 would be confirmed by greater AIC activation during the performance of intrinsically, relative to non-intrinsically, motivating tasks, during both question answering and anagram solving. H1 would be further confirmed by significant correlations between participants' self-reported trial-by-trial task interest and AIC activations while performing the intrinsically motivating tasks. That is, AIC activity was predicted to occur (1) during the performance of intrinsically motivating tasks and (2) during the subjective feeling of high task interest.

We also hypothesized that the activation of brain regions related to reward processing (i.e., striatum) would be related to the experimentally manipulated high versus low intrinsic actual experience of intrinsic motivation ( $\mathrm{H} 2)$. $\mathrm{H} 2$ would be confirmed by greater striatum activation during the performance of intrinsically, relative to non-intrinsically, motivating tasks.

Finally, the especially new and important aspect of in the present study is that we further hypothesized that intrinsicsatisfaction-related AIC activity and reward-processing- 
related striatum activity would show increased functional interactions in the intrinsic-motivation system (H3). The basis of this prediction was the proposition that the AIC and striatum interact - or work together during the experience of intrinsic motivation - to constitute the intrinsic-motivation system. We based $\mathrm{H} 3$ on the well-known anatomical and functional connectivity between the AIC and striatum in the motivation circuitry (Chikama, McFarland, Amaral, \& Haber, 1997; Cho et al., 2013). H3 would be confirmed by increased AIC-striatum interaction during the performance of the intrinsically, relative to non-intrinsically, motivating tasks.

\section{Method}

\section{Participants}

Twenty-two undergraduates (11 females, 11 males; mean age $22.9 \pm 2.87$ years), recruited from a large private university in Korea, participated in the functional magnetic resonance imaging (fMRI) study. Each participant was right-handed and had normal or corrected-to-normal vision and no history of neurological illness. The participants provided informed consent and received compensation for their participation. This study was approved by the institutional review board of Korea University. Before we collected our data, we computed a power analysis for a two-group independent $t$ test (G*Power 3; Faul, Erdfelder, Lang, \& Buchner, 2007) to determine the appropriate sample size. Using $p=.05$ and an expected effect size of $d=1.80$ (based on a threshold $t$ ratio of 4.00), we calculated that the sample size needed to obtain a power of .95 was 20 , which was similar to the sample sizes used in previous neuroscientific investigations of intrinsic motivation (Gruber et al., 2014; Kang et al., 2009).

\section{Experimental tasks}

Because novelty/challenge seeking and subsequent curiosity/ competence satisfaction are the defining characteristics of intrinsic motivation, two types of tasks were used: curiosityinducing versus non-curiosity-inducing question answering (Table 1), and competence-enabling versus non-competenceenabling anagram solving (Table 2).

Curiosity-inducing questions asked about new information in various domains (e.g., What animal can shed up to 30,000 teeth in its lifetime?), which were adopted from Kang and her colleagues' (2009) study and from trivia quiz books. In contrast, non-curiosity-inducing questions asked about alreadyknown information (e.g., What country is the world's most populous?), which were obtained from general-knowledge quiz books and high-school textbooks. Upon seeing each question, participants were asked to think of the correct answer, and participants knew that the correct answer would be provided a few seconds later. For this operational definition of high versus low curiosity-inducing stimuli, we relied on Lowenstein's (1994) information-gap perspective on curiosity. According to this perspective, when people are asked a question about information they already know, no information gap in their existing knowledge is revealed, and they do not anticipate any pleasure or satisfaction from attaining the nonmissing information. In contrast, when people are asked a question about information they do not know, the question alerts them to the existence of a suddenly salient information gap in their knowledge, and they do anticipate pleasure and satisfaction upon attaining the missing information. Constructed in this way, the curiosity-inducing questions could be expected to yield greater intrinsic motivation because they offered opportunities to discover new information, to build suspense about what would happen next, and to solve a mystery - that is, to resolve a gap in their knowledge and, hence, experience satisfaction from a feeling of knowing (Abuhamdeh et al., 2015; Deci \& Ryan, 1985; Loewenstein, 1994; Silvia, 2008). The sentence structures and lengths (in number of words) for the curiosity-inducing and noncuriosity-inducing questions were matched to be equivalent. For the present study, we selected 21 matched pairs of curiosity-inducing and non-curiosity-inducing questions based on the results of a pilot test (see the Pilot Test Results section below).

Competence-enabling and non-competence-enabling anagrams were developed on the basis of the experimental task of a previous study (Reeve, 1989). Upon seeing each five-letter anagram, participants were asked to unscramble the five jumbled letters to produce a familiar word. The competenceenabling anagrams utilized difficult-to-decode letter patterns (e.g., "OHTMN" transposed from "MONTH"), whereas the non-competence-enabling anagrams utilized easy-to-decode letter patterns (e.g., "CLCOK" transposed from "CLOCK"). To ensure that the former anagrams were competenceenabling and interesting, rather than frustrating and too difficult (given the 7-s time limit), each anagram trial included a first-two-letters hint in the middle of its problem presentation (e.g., $\mathrm{MO}_{-}{ }_{-}$and $\mathrm{CL}_{--}$, for the two examples above). We provided participants with the first-two-letter hint at the 3-s mark because pilot testing had shown that the anagram solution rate was low with no hints, but very high with a first-twoletter hint, thereby assuring that participants were exposed to both optimal challenge (from the difficult-to-decode five-letter anagram) and a competence-satisfying mastery experience (enabled by the first-two-letter hint) (Clifford, 1990; Kawabata \& Mallett, 2011). The lengths (in number of letters) and hint provisions (correct first two letters) for the competence-enabling and non-competence-enabling anagrams were matched to be equivalent. For the present study, we selected 21 matched pairs of competence-enabling and non-competence-enabling anagrams based on the results of a pilot test (see the Pilot Test Results section below). 
Table 1 Examples of the curiosity-inducing and non-curiosity-inducing questions

\begin{tabular}{|c|c|c|c|c|}
\hline & Curiosity-Inducing Questions & Answers & Non-Curiosity-Inducing Questions & Answers \\
\hline 1 & What animal can shed up 30,000 teeth in its lifetime? & Shark & What country is the world's most populous? & China \\
\hline 2 & What book is the most shoplifted book? & The Bible & $\begin{array}{l}\text { What is the official currency of the United } \\
\text { States? }\end{array}$ & US dollar \\
\hline 3 & What instrument was invented to sound like a human singing? & Violin & What does a red traffic light mean? & Stop \\
\hline 4 & What was the first animated film to win an Academy Award? & $\begin{array}{l}\text { Beauty and } \\
\text { the Beast }\end{array}$ & $\begin{array}{l}\text { Which company manufactured iPhone } \\
\text { and iPad? }\end{array}$ & Apple \\
\hline 5 & Which continent is divided into the most countries? & Africa & What is the capital city of France? & Paris \\
\hline
\end{tabular}

First four curiosity-inducing question examples were adopted from Kang and her colleagues' (2009) study

\section{Pilot test results}

After conducting preliminary pilot work to develop the curiosity-inducing questions and the competence-enabling anagrams, we conducted further pilot tests to validate the intrinsic-motivation-generating capacity of these stimuli. In Pilot Test 1, 14 undergraduates (seven females, seven males; mean age $24.5 \pm 2.53$ years) from the same university viewed the curiosity-inducing and non-curiosity-inducing questions and rated how suspenseful, curiosity satisfying, interesting, and difficult to solve each question was on a scale from 1 (not at all) to 5 (a great deal). Participants' ratings for these four dependent measures, broken down by experimental condition, appear in Fig. 1A. The suspense, curiosity satisfaction, interest, and difficulty ratings were all significantly greater for the curiosity-inducing questions than for the non-curiosityinducing questions, $t \mathrm{~s}(13) \geq 10.38, p \mathrm{~s}<.001$, confirming that the non-curiosity-inducing questions represented a bland version of the question-answering task, whereas the curiosityinducing questions represented an intrinsically motivating version (i.e., suspenseful, satisfying, and interesting) of the same task. We assessed the difficulty ratings to control for this possible stimulus confound in the main analyses.

In Pilot Test 2, 12 undergraduates (seven females, five males; mean age $22.7 \pm 3.68$ years) from the same university solved the competence-enabling versus non-competence-

Table 2 Examples of the competence-enabling and non-competenceenabling anagrams

\begin{tabular}{lllll}
\hline $\begin{array}{l}\text { Competence- } \\
\text { Enabling } \\
\text { Anagrams }\end{array}$ & Answers & $\begin{array}{l}\text { Non-Competence- } \\
\text { Enabling } \\
\text { Anagrams }\end{array}$ & Answers \\
\hline 1 & OHTMN & MONTH & CLCOK & CLOCK \\
2 & CRPEI & PRICE & WHTIE & WHITE \\
3 & NRITA & TRAIN & HAYPP & HAPPY \\
4 & HUOCG & COUGH & IFRST & FIRST \\
5 & SPEUA & PAUSE & THIKN & THINK \\
\hline
\end{tabular}

enabling anagrams and rated how optimally challenging, competence satisfying, interesting, and difficult to solve each anagram was on the same 5-point scale. Participants' ratings for these four dependent measures, broken down by experimental condition, appear in Fig. 1B. The challenge, competence satisfaction, interest, and difficulty ratings were all significantly greater for the competence-enabling anagrams than for the non-competence-enabling anagrams, $t \mathrm{~s}(11) \geq 4.89, p \mathrm{~s}<$ .001 , confirming that the non-competence-enabling anagrams represented a bland version of the anagram-solving task, whereas the competence-enabling anagrams represented an intrinsically motivating version (i.e., challenging, satisfying, and interesting) of the same task. Again we assessed the difficulty ratings to control for this possible stimulus confound in the main analyses.

\section{Procedure}

An event-related fMRI experiment, which consisted of three separate runs, was performed. Each run lasted $8 \mathrm{~min}$ and consisted of 28 trials (i.e., seven trials each for the curiosityinducing questions, non-curiosity-inducing questions, competence-enabling anagrams, and non-competenceenabling anagrams). In each trial (Fig. 2), one randomly selected question or anagram was presented. For the curiosityinducing and non-curiosity-inducing questions, only the question was presented for $7 \mathrm{~s}$. For the competence-enabling and non-competence-enabling anagrams, only the anagram was presented for $3 \mathrm{~s}$, and then the hint was added and presented together with the anagram for $4 \mathrm{~s}$. During each 7 -s presentation, participants were asked to think of the correct answer before it was provided. Then the correct answer was given for $3 \mathrm{~s}$. After a 1-s fixation was presented, participants were asked to rate how interesting the question or anagram was on a 1 (not at all) to 4 (a great deal) scale for 2 s. Participants were asked to press one of the four buttons using their right hand in order to make this series of interest ratings. Before the next trial began, an intertrial interval was presented for an average of $4 \mathrm{~s}(2,000-6,000 \mathrm{~ms})$. The trial order and intertrial interval 


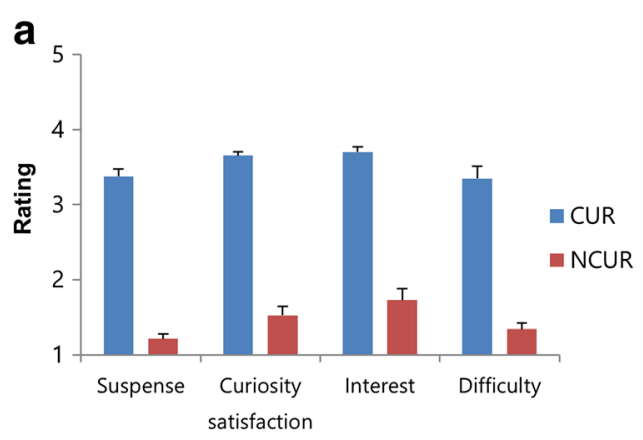

Fig. 1 Participants' mean suspense, curiosity satisfaction, interest, and difficulty ratings for curiosity-inducing and non-curiosity-inducing questions are presented (A). The curiosity-inducing questions were rated as being significantly more suspenseful, curiosity satisfying, interesting, and difficult than the non-curiosity-inducing questions. Participants' mean challenge, competence satisfaction, interest, and difficulty ratings for competence-enabling and non-competence-enabling anagrams are

variation were determined using OptSeq (http://surfer.nmr. mgh.harvard.edu/optseq/).

Before the fMRI scanning, participants received the task instructions. During the fMRI scanning, functional images were acquired while participants performed the experimental task, and structural images were then obtained. After the experiment was over, participants were debriefed.

\section{fMRI data acquisition}

A 3-T Trio MRI scanner (Siemens, Erlangen, Germany) was used for functional and structural imaging. A series of 32-slice functional images were acquired using a $\mathrm{T} 2 *$-weighted gradient-echo echo-planar imaging (EPI) sequence sensitive to a blood-oxygenation-level-dependent (BOLD) contrast. The following parameters were used for the functional images: $\mathrm{TR}=2,000 \mathrm{~ms}, \mathrm{TE}=30 \mathrm{~ms}$, flip angle $=90^{\circ}$, field of

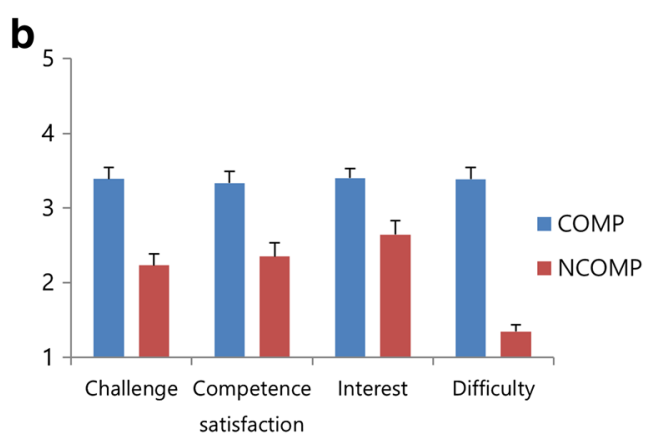

also presented (B). The competence-enabling anagrams were rated as being significantly more optimally challenging, competence satisfying, interesting, and difficult than the non-competence-enabling anagrams. CUR: curiosity-inducing questions, NCUR: non-curiosity-inducing questions, COMP: competence-enabling anagrams, NCOMP: non-competence-enabling anagrams

view $=224 \times 224$, in-plane resolution $=3.5 \times 3.5 \mathrm{~mm}$, and slice thickness $=4 \mathrm{~mm}$ with no gap. After we had obtained the functional images, high-resolution T1-weighted structural images were acquired by using an MP-RAGE sequence with the following parameters: $\mathrm{TR}=1,900 \mathrm{~ms}$, $\mathrm{TE}=2.52 \mathrm{~ms}$, flip angle $=9^{\circ}$, field of view $=256 \times 256$, in-plane resolution $=$ $1 \times 1 \mathrm{~mm}$, and slice thickness $=1 \mathrm{~mm}$ with no gap. The structural images were used for anatomical localization in order to facilitate precise determination of the structures corresponding to the functional activation foci.

\section{fMRI data analysis}

The brain images were analyzed by using the AFNI software (Cox, 1996; http://afni.nimh.nih.gov). The first three images of each run were discarded in order to allow the hemodynamics and MRI signals to reach a steady state. In preprocessing, the

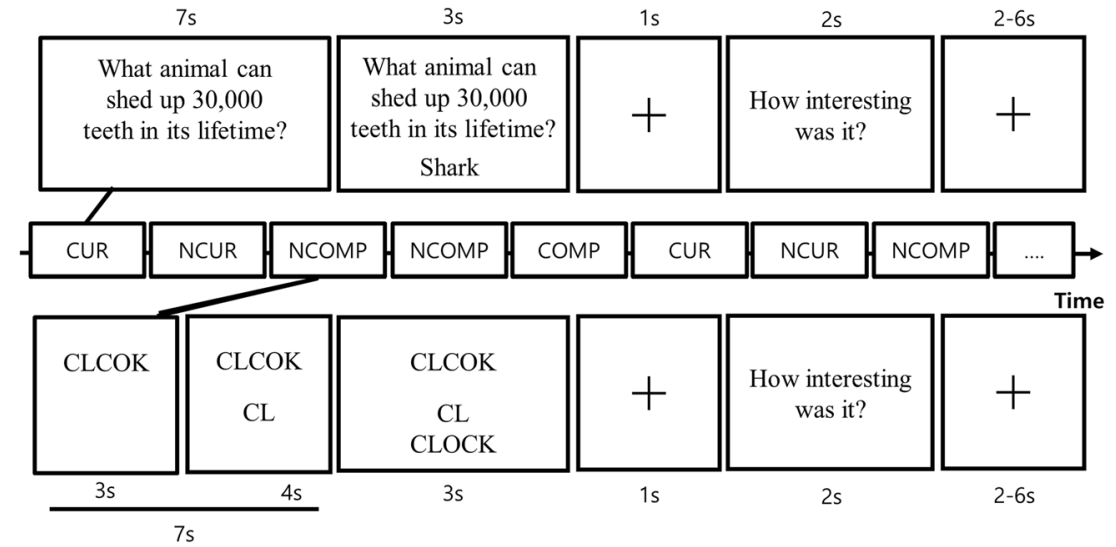

Fig. 2 Experimental task and experimental design. In each trial, one randomly selected question or anagram was presented for $7 \mathrm{~s}$, during which participants were asked to think of the correct answer before it was provided. For anagrams, after $3 \mathrm{~s}$ had passed, the hint was added for $4 \mathrm{~s}$. After a 1-s fixation was presented, participants were asked to rate how interesting the question or anagram had been. CUR: curiosityinducing questions, NCUR: non-curiosity-inducing questions, COMP: competence-enabling anagrams, NCOMP: non-competence-enabling anagrams 
functional images were interpolated to the same time point at the beginning of the TR for temporal alignment. Then, the temporally aligned functional images were registered to the structural images of each participant for spatial alignment and registered to the base volume of the functional images for head motion correction. The spatially realigned functional images were spatially blurred with a $5-\mathrm{mm}$ full-width at halfmaximum (FWHM) Gaussian kernel. After the values of voxels outside the brain were excluded, the functional data were normalized as percentages of the mean to conduct the statistical analyses.

In individual-level analyses, each participant's preprocessed time series data were analyzed using a general linear model (GLM) with 14 regressors convoluted with hemodynamic response functions (HRF). Four regressors were included for the time periods that correct answers were presented (i.e., the moment that curiosity or challenge, if it existed, was satisfied) in the two experimental conditions (i.e., curiosity-inducing questions, competence-enabling anagrams) and in the two corresponding control conditions (i.e., non-curiosity-inducing questions, noncompetence-enabling anagrams). Four regressors were included for the exact time points that participants responded to the interest ratings in the experimental and control conditions, to control for the effects of motor artifacts. Six regressors were included for head motion parameters, to control for the effects of head motion artifacts. Each participant's statistical data were transformed so as to fit the Montreal Neurological Institute (MNI) template using her or his standardized high-resolution structural images and were resampled to $2 \times 2 \times 2 \mathrm{~mm}^{3}$ voxels.

In the group-level analyses, subtraction analysis was first conducted to identify the neural substrates when participants performed intrinsically motivating tasks (i.e., curiosityinducing questions, competence-enabling anagrams) as compared to when they performed non-intrinsically-motivating tasks (i.e., non-curiosity-inducing questions, non-competenceenabling anagrams). Subtraction analyses comparing the neural differences between curiosity-inducing and non-curiosityinducing questions and between competence-enabling and non-competence-enabling anagrams were then conducted separately as supplemental analyses. For correcting multiple comparison inferences in the subtraction analyses, the Monte Carlo simulation method (Forman et al., 1995) was used, which determined the cluster-wise threshold (corrected $p<.05$ ) considering both the voxel-wise threshold $(p<.001)$ and cluster size $\left(n \geq 25\right.$, a minimum volume of $\left.200 \mathrm{~mm}^{3}\right){ }^{1}$

\footnotetext{
${ }^{1}$ Considering "the balance between Type I and Type II error rates" (Lieberman \& Cunningham, 2009), we preferred using a relatively liberal voxel-wise threshold and a larger minimum cluster size (e.g., $p<.005$ with at least 52 contiguous voxels) to using a conservative voxel-wise threshold and smaller minimum cluster size (e.g., $p<.001$ with at least 25 contiguous voxels), which possibly simplifies such phenomena as publishing "large and obvious effects." For spatial specificity, however, we used the conservative voxel-wise threshold and smaller minimum cluster size for the subtraction analyses of this study.
}

To control for the possible confounding effects of task difficulty, a supplemental subtraction analysis was conducted-namely, the neural activations during the performance of intrinsically motivating tasks were compared to those during the performance of non-intrinsically-motivating tasks after controlling for trial-specific task difficulty. For this analysis, each participant's preprocessed data were analyzed using another GLM considering the difficulty level of each question and anagram, obtained from the pilot test results (recall Fig. 1), as a covariate parameter. The only difference between this GLM and the original one was that the regressors for the experimental and control conditions were modulated by the task difficulty rating for each question or anagram. The individual-level data based on this GLM allowed us to separate the neural activations of each condition, with task difficulty effects partialed out, from the neural activations correlated with the task difficulty of each condition. In the group-level analysis, we conducted the subtraction analysis by directly contrasting the neural activity of the intrinsically motivating tasks versus the neural activity of the non-intrinsically-motivating tasks, using the individual-level data with task difficulty effects partialed out. To correct for multiple comparison inferences in the subtraction analysis, we used the cluster-wise threshold (correct $p<.05$ ), determined by the voxel-wise threshold $(p<.001)$ and the cluster size ( $n \geq 25$, a minimum volume of $200 \mathrm{~mm}^{3}$ ).

We conducted a parametric analysis to identify the brain regions correlated with the degree of participants' trial-bytrial self-reported interest during the performance of intrinsically motivating tasks. For this analysis, each participant's preprocessed data were analyzed using another GLM considering the participants' ongoing interest ratings on all curiosity-inducing questions and competence-enabling anagrams as modulating parameters. In this GLM, the regressors for the experimental and control conditions were modulated by each participant's interest rating for each question or anagram. The individual-level data based on this GLM allowed us to separate the neural activations modulated by participants' interest in each condition from the neural activations of each condition, with interest effects partialed out. In the group-level analysis, we conducted a parametric analysis on the individual-level data modulated by participants' interest to identify the brain regions correlated with the degree of participants' interest during performance of the intrinsically motivating tasks. For correcting multiple comparison inferences in the parametric analysis, we used the cluster-wise threshold (correct $p<.05$ ), determined by the voxel-wise threshold $(p<.005)$ and the cluster size $(n \geq 52$, a minimum volume of $416 \mathrm{~mm}^{3}$ ).

To understand the neural interaction patterns of intrinsic motivation, psychophysiological interaction (PPI; Friston et al., 1997) analyses were performed. Because the striatum is a well-known region for human motivation (Delgado, 2007; 
Haber \& Knutson, 2010; O’Doherty, 2004), the regions of left and right striatum activated in the subtraction analysis between intrinsically motivating tasks versus non-intrinsicallymotivating tasks were chosen as the seed brain regions for the PPI analyses. For these PPI analyses, each participant's preprocessed data were analyzed using another GLM. In this GLM, the regressors for the neural responses of the striatum and its neural interactions with each of the experimental and control conditions were additionally included, as well as the regressors considered in the original GLM. In the group-level analyses, we examined the neural interactions of the left and right striatum with other brain regions during the performance of intrinsically motivating as compared to non-intrinsicallymotivating tasks. To correct for multiple comparison inferences in the PPI analyses, we used the cluster-wise threshold (correct $p<.05$ ), determined by the voxel-wise threshold ( $p<$ $.005)$ and the cluster size ( $n \geq 52$, a minimum volume of 416 $\mathrm{mm}^{3}$ ).

The brain regions significantly activated in the group-level analyses were reported in MNI coordinates. Regions of interest (ROIs) were set from the brain regions significantly activated in these analyses, and the BOLD signal changes in these ROIs were compared across conditions to understand the neural activation differences. To avoid the issue of nonindependence bias (Kriegeskorte, Simmons, Bellgowan, \& Baker, 2009), a leave-one-subject-out (LOSO) method (Esterman, Tamber-Rosenau, Chiu, \& Yantis, 2010) was used in the analyses of ROIs. That is, we left out one participant's individuallevel data, ran 22 independent group-level analyses using only the 21 remaining participants' individual-level data to define the independent clusters of the ROIs, and extracted the BOLD signal changes in these ROIs from the left-out participants' data.

\section{Results}

\section{Behavioral results}

To complete a manipulation check in the main study to confirm the intrinsic-motivation-generating capacity of the stimuli, participants made interest ratings on a $1-4$ scale (i.e., How interesting was it?) following each curiosity-inducing, non-curiosity-inducing, competence-enabling, and non-competenceenabling trial. The curiosity-inducing questions $(M=3.08 \pm$ $0.08)$ and competence-enabling anagrams $(M=2.35 \pm 0.09)$ were rated as being significantly more interesting (i.e., significantly more intrinsically motivating) than the non-curiosityinducing questions $(M=1.64 \pm 0.12)$ and non-competenceenabling anagrams $(M=1.87 \pm 0.12)$, respectively: $t(21)=$ $11.11, p<.001$, for curiosity-inducing versus non-curiosityinducing questions; $t(21)=6.56, p<.001$, for competenceenabling versus non-competence-enabling anagrams.

\section{fMRI results}

The results of the subtraction analysis (Table 3) revealed that both bilateral AIC (Fig. 3A) and striatum (Fig. 3B) were more activated when participants performed intrinsically motivating tasks (i.e., curiosity-inducing questions, competence-enabling anagrams) than when they performed non-intrinsically-motivating tasks (i.e., non-curiosityinducing questions, non-competence-enabling anagrams) ( $p$ $<.001$ with at least 25 contiguous voxels; corrected $p<$ $.05)$. BOLD signal changes in the ROIs (i.e., AIC, striatum) were compared across conditions, which confirmed greater activity of bilateral AIC and striatum during the performance of intrinsically motivating tasks than during the performance of non-intrinsically-motivating tasks (Fig. 3). In addition to these hypothesized brain regions, the left thalamus, bilateral cerebellum, bilateral dorsolateral prefrontal cortex (DLPFC), right medial frontal gyrus, left precentral gyrus, left angular gyrus, and right occipital lobe were also more activated when participants performed intrinsically motivating tasks than when they performed nonintrinsically-motivating tasks $(p<.001$ with at least 25 contiguous voxels; corrected $p<.05)$.

The results of a subtraction analysis broken down specifically by curiosity-inducing versus non-curiosity-inducing questions appear in Table 4, whereas the results of a subtraction analysis broken down specifically by competenceenabling versus non-competence-enabling anagrams appear in Table 5. The important point within these supplemental analyses is that the AIC and striatum were both more activated on both the curiosity-inducing questions and the competenceenabling anagrams.

The results of the subtraction analysis while controlling for the effects of task difficulty (see Table 6) showed very similar neural activation patterns, as can be seen by a comparison of the results in Table 3 with those in Table 6. In particular, the $\mathrm{AIC}$ and striatum consistently showed greater activation during the performance of intrinsically motivating tasks than during the performance of non-intrinsically-motivating tasks, even when controlling for the effects of the difficulty levels of all questions and anagrams $(p<.001$ with at least 25 contiguous voxels; corrected $p<.05){ }^{2}$

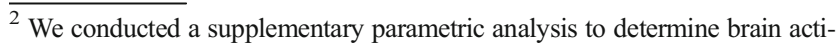
vations that covaried with task difficulty. As the difficulty level of each curiosity-inducing question or competence-enabling anagram increased, the left occipital lobe (peak MNI coordinate: $-20,-84,-12$; maximum $t$ value $=$ 3.66; volume: $856 \mathrm{~mm}^{3}$ ) showed greater activations during the performance of intrinsically motivating tasks, whereas the right precuneus (peak MNI coordinate: $6,-68,24$; maximum $t$ value $=4.16$; volume: $1,312 \mathrm{~mm}^{3}$ ) and left paracentral lobe (peak MNI coordinate: $-2,-20,52$; maximum $t$ value $=$ 4.20; volume: $\left.688 \mathrm{~mm}^{3}\right)$ showed greater deactivations $(p<.005$ with at least 52 contiguous voxels; corrected $p<.05$ ). These analyses are important because they show that the neural activation patterns associated with task difficulty were not similar to those associated with intrinsically motivating as compared to non-intrinsically motivating tasks and with self-reported interest ratings.
} 
Table 3 Results of the subtraction analyses between intrinsically motivating task conditions and non-intrinsically-motivating task conditions

\begin{tabular}{|c|c|c|c|c|c|c|c|}
\hline \multirow[t]{2}{*}{ Brain Region } & \multirow[t]{2}{*}{ BA } & \multirow[t]{2}{*}{ Volume } & \multirow[t]{2}{*}{ Side } & \multicolumn{3}{|c|}{ MNI Coordinates } & \multirow[t]{2}{*}{ Maximum $t$ Value } \\
\hline & & & & $x$ & $y$ & $z$ & \\
\hline \multicolumn{8}{|c|}{ (Curiosity-Inducing Questions + Competence-Enabling Anagrams) - (Non-Curiosity-Inducing Questions + Non-Competence-Enabling Anagrams } \\
\hline $\begin{array}{l}\text { Dorsolateral prefrontal cortex \& Anterior } \\
\text { insular cortex }{ }^{\text {a }}\end{array}$ & $9 / 13$ & 23,920 & $\mathrm{~L}$ & -50 & 6 & 24 & 8.90 \\
\hline Anterior insular cortex & 13 & 2,584 & $\mathrm{R}$ & 30 & 24 & -2 & 6.99 \\
\hline \multirow[t]{2}{*}{ Striatum } & & 656 & $\mathrm{R}$ & 10 & -2 & -8 & 4.87 \\
\hline & & 608 & $\mathrm{~L}$ & -20 & 2 & 8 & 6.21 \\
\hline Thalamus & & 2,304 & $\mathrm{~L}$ & -10 & -12 & 4 & 6.83 \\
\hline \multirow[t]{2}{*}{ Cerebellum } & & 27,176 & $\mathrm{R}$ & 32 & -64 & -30 & 9.70 \\
\hline & & 240 & $\mathrm{~L}$ & -4 & -52 & -38 & 4.77 \\
\hline Dorsolateral prefrontal cortex & 46 & 4,688 & $\mathrm{R}$ & 46 & 28 & 14 & 7.71 \\
\hline Medial frontal gyrus & 32 & 9,680 & $\mathrm{R}$ & 2 & 14 & 42 & 8.96 \\
\hline Precentral gyrus & 6 & 784 & $\mathrm{~L}$ & -30 & -2 & 44 & 6.24 \\
\hline Angular gyrus & 39 & 5,576 & $\mathrm{~L}$ & -30 & -62 & 38 & 7.79 \\
\hline Occipital lobe & 18 & 16,192 & $\mathrm{R}$ & -22 & -90 & -8 & 9.70 \\
\hline
\end{tabular}

The cluster-wise threshold (correct $p<.05$ ) was determined by the voxel-wise threshold $(p<.001)$ and the cluster size $(n \geq 25$, a minimum volume of 200 $\left.\mathrm{mm}^{3}\right) .{ }^{\text {a }}$ The activations of the dorsolateral prefrontal cortex and anterior insular cortex were not observed separately at this cluster-wise threshold. However, these activations were separable, and both were statistically significant at the conservative voxel-wise threshold $(p<.00005)$ and smaller minimum volume $\left(n \geq 9\right.$, a minimum volume of $\left.72 \mathrm{~mm}^{3}\right)$ that corresponded to corrected $p<.05$
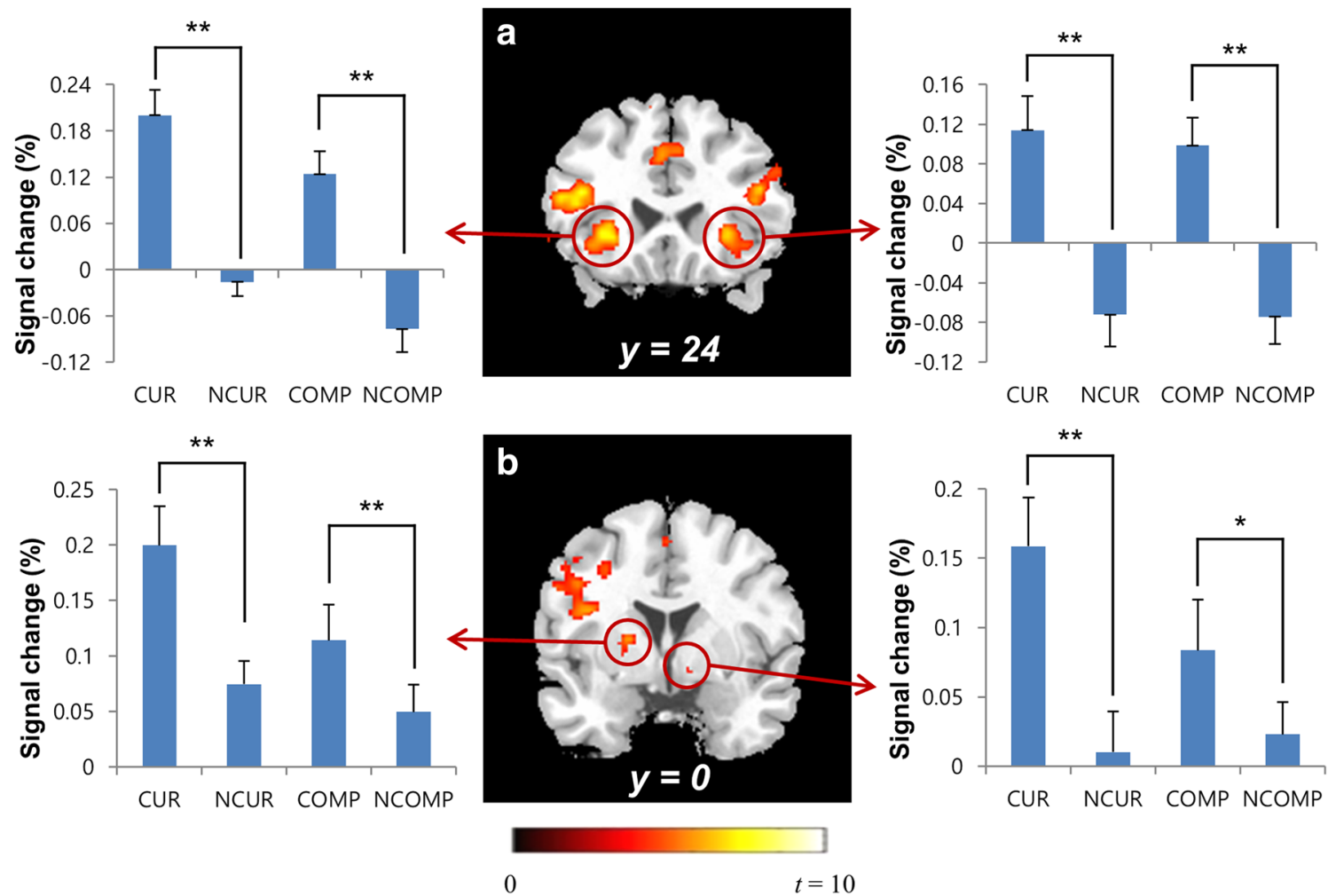

Fig. 3 Bilateral anterior insular cortex (AIC) (A) and striatum (B) were more activated during the performance of intrinsically motivating tasks than during the performance of non-intrinsically-motivating tasks $(p<$ .001 with at least 25 contiguous voxels; corrected $p<.05$ ). BOLD signal changes across the conditions are presented. For spatial specificity,

BOLD signal changes in the left AIC were extracted from the activations observed at the conservative voxel-wise threshold $(p<.00005) .{ }^{*} p<.05$, ${ }^{* *} p<.01$. CUR: curiosity-inducing questions, NCUR: non-curiosityinducing questions, COMP: competence-enabling anagrams, NCOMP: non-competence-enabling anagrams 
Table 4 Results of the subtraction analysis between the curiosity-inducing and non-curiosity-inducing conditions

\begin{tabular}{|c|c|c|c|c|c|c|c|}
\hline \multirow[t]{2}{*}{ Brain Region } & \multirow[t]{2}{*}{$\mathrm{BA}$} & \multirow[t]{2}{*}{ Volume } & \multirow[t]{2}{*}{ Side } & \multicolumn{3}{|c|}{ MNI Coordinates } & \multirow[t]{2}{*}{ Maximum $t$ Value } \\
\hline & & & & $x$ & $y$ & $z$ & \\
\hline \multicolumn{8}{|c|}{ Curiosity-Inducing Questions - Non-Curiosity-Inducing Questions } \\
\hline $\begin{array}{l}\text { Dorsolateral prefrontal cortex \& Anterior } \\
\text { insular cortex }{ }^{\text {a }}\end{array}$ & $9 / 13$ & 17,512 & $\mathrm{~L}$ & -48 & 14 & 24 & 9.13 \\
\hline Anterior insular cortex & 13 & 752 & $\mathrm{R}$ & 30 & 22 & -8 & 5.95 \\
\hline \multirow[t]{2}{*}{ Striatum } & & 4,520 & $\mathrm{R}$ & 8 & -2 & -6 & 6.64 \\
\hline & & 624 & $\mathrm{~L}$ & -18 & 0 & -6 & 6.08 \\
\hline Cerebellum & & 6,952 & $\mathrm{R}$ & 30 & -62 & -32 & 8.80 \\
\hline Dorsolateral prefrontal cortex & 46 & 344 & $\mathrm{R}$ & 48 & 28 & 16 & 4.85 \\
\hline Medial frontal gyrus & 32 & 6,952 & $\mathrm{~L}$ & -4 & 18 & 42 & 6.93 \\
\hline Superior frontal gyrus & 8 & 1,360 & $\mathrm{~L}$ & -6 & 38 & 50 & 6.26 \\
\hline Inferior frontal gyrus & 44 & 672 & $\mathrm{R}$ & 38 & 10 & 22 & 4.94 \\
\hline \multirow[t]{2}{*}{ Middle temporal gyrus } & 20 & 856 & $\mathrm{~L}$ & -58 & -36 & -14 & 5.38 \\
\hline & 21 & 664 & $\mathrm{~L}$ & -56 & -6 & -14 & 5.66 \\
\hline Superior temporal gyrus & 38 & 288 & $\mathrm{~L}$ & -44 & 20 & -26 & 4.87 \\
\hline Angular gyrus & 39 & 312 & $\mathrm{~L}$ & -32 & -62 & 32 & 5.47 \\
\hline \multirow[t]{2}{*}{ Occipital lobe } & 17 & 4,648 & $\mathrm{~L}$ & -18 & -94 & -12 & 8.02 \\
\hline & 17 & 4,152 & $\mathrm{R}$ & 20 & -98 & -4 & 5.98 \\
\hline
\end{tabular}

The cluster-wise threshold (correct $p<.05)$ was determined by the voxel-wise threshold $(p<.001)$ and the cluster size $\left(25\right.$ contiguous voxels; $\left.200 \mathrm{~mm}^{3}\right)$.

${ }^{a}$ The activations of the dorsolateral prefrontal cortex and anterior insular cortex were not observed separately at this cluster-wise threshold. However, these activations were separable, and both were statistically significant at the conservative voxel-wise threshold $(p<.00005)$ and smaller minimum volume (nine contiguous voxels; $72 \mathrm{~mm}^{3}$ ) that corresponded to corrected $p<.05$

Table 5 Results of the subtraction analysis between the competence-enabling and non-competence-enabling conditions

\begin{tabular}{|c|c|c|c|c|c|c|c|}
\hline \multirow[t]{2}{*}{ Brain Region } & \multirow[t]{2}{*}{$\mathrm{BA}$} & \multirow[t]{2}{*}{ Volume } & \multirow[t]{2}{*}{ Side } & \multicolumn{3}{|c|}{ MNI Coordinates } & \multirow{2}{*}{$\begin{array}{l}\text { Maximum } \\
t \text { Value }\end{array}$} \\
\hline & & & & $x$ & $y$ & $z$ & \\
\hline \multicolumn{8}{|c|}{ Competence-Enabling Anagrams - Non-Competence-Enabling Anagrams } \\
\hline $\begin{array}{l}\text { Anterior insular cortex \& Dorsolateral } \\
\text { prefrontal cortex }{ }^{\text {a }}\end{array}$ & $13 / 9$ & 20,864 & $\mathrm{~L}$ & -32 & 20 & -4 & 8.74 \\
\hline Anterior insular cortex & 13 & 2,560 & $\mathrm{R}$ & 40 & 20 & -8 & 7.45 \\
\hline Striatum & & 632 & $\mathrm{~L}$ & -20 & 0 & -6 & 4.67 \\
\hline Thalamus & & 656 & $\mathrm{~L}$ & -10 & -12 & 4 & 6.13 \\
\hline Cerebellum & & 14,824 & $\mathrm{R}$ & 4 & -72 & -30 & 7.28 \\
\hline \multirow[t]{3}{*}{ Dorsolateral prefrontal cortex } & 46 & 3,792 & $\mathrm{R}$ & 48 & 34 & 18 & 7.25 \\
\hline & 9 & 976 & $\mathrm{R}$ & 48 & 10 & 26 & 4.55 \\
\hline & 9 & 256 & $\mathrm{R}$ & 54 & 10 & 38 & 5.37 \\
\hline Medial frontal gyrus & 32 & 6,320 & $\mathrm{~L}$ & -4 & 16 & 38 & 9.59 \\
\hline Middle frontal gyrus & 6 & 264 & $\mathrm{R}$ & 40 & 4 & 54 & 4.98 \\
\hline Precentral gyrus & 6 & 848 & $\mathrm{~L}$ & -30 & -4 & 40 & 4.82 \\
\hline \multirow[t]{2}{*}{ Precuneus } & 19 & 10,728 & $\mathrm{~L}$ & -30 & -64 & 44 & 9.22 \\
\hline & 19 & 3,128 & $\mathrm{R}$ & 24 & -68 & 42 & 5.63 \\
\hline Occipital lobe & 19 & 22,496 & $\mathrm{~L}$ & -50 & -70 & -16 & 8.65 \\
\hline
\end{tabular}

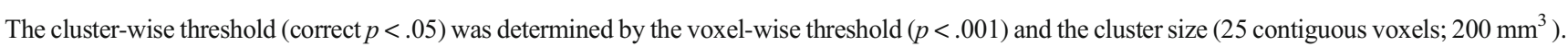

a The activations of the dorsolateral prefrontal cortex and anterior insular cortex were not observed separately at this cluster-wise threshold. However, these activations were separable, and both were statistically significant at the conservative voxel-wise threshold $(p<.0001)$ and smaller minimum volume (11 contiguous voxels; $88 \mathrm{~mm}^{3}$ ) that corresponded to corrected $p<.05$ 
Table 6 Results of the subtraction analysis between intrinsically motivating task conditions and non-intrinsically-motivating task conditions, controlling for the effects of task difficulty

\begin{tabular}{|c|c|c|c|c|c|c|c|}
\hline \multirow[t]{2}{*}{ Brain Region } & \multirow[t]{2}{*}{ BA } & \multirow[t]{2}{*}{ Volume } & \multirow[t]{2}{*}{ Side } & \multicolumn{3}{|c|}{ MNI Coordinates } & \multirow[t]{2}{*}{ Maximum $t$ Value } \\
\hline & & & & $x$ & $y$ & $z$ & \\
\hline \multicolumn{8}{|c|}{ (Curiosity-Inducing Questions + Competence-Enabling Anagrams) $-($ Non-Curiosity-Inducing Questions + Non-Competence-Enabling Anagram } \\
\hline $\begin{array}{l}\text { Dorsolateral prefrontal cortex \& Anterior } \\
\text { insular cortex }{ }^{\text {a }}\end{array}$ & $9 / 13$ & 47,160 & $\mathrm{~L}$ & -50 & 6 & 24 & 9.58 \\
\hline Anterior insular cortex & 13 & 3,888 & $\mathrm{R}$ & 30 & 24 & -2 & 8.11 \\
\hline Striatum & & 1,696 & $\mathrm{R}$ & 10 & -2 & -8 & 5.81 \\
\hline \multirow[t]{2}{*}{ Cerebellum } & & 53,280 & $\mathrm{R}$ & 30 & -58 & -32 & 9.39 \\
\hline & & 472 & $\mathrm{~L}$ & -4 & -52 & -38 & 5.16 \\
\hline \multirow[t]{2}{*}{ Dorsolateral prefrontal cortex } & 46 & 8,888 & $\mathrm{R}$ & 46 & 28 & 14 & 7.92 \\
\hline & 9 & 344 & $\mathrm{R}$ & 54 & 8 & 40 & 5.01 \\
\hline Medial frontal gyrus & 32 & 14,080 & $\mathrm{~L}$ & -2 & 16 & 40 & 9.96 \\
\hline Middle frontal gyrus & 6 & 416 & $\mathrm{R}$ & 38 & 4 & 54 & 5.33 \\
\hline Middle temporal gyrus & 21 & 216 & $\mathrm{~L}$ & -48 & -42 & -6 & 4.21 \\
\hline Angular gyrus & 39 & 9,168 & $\mathrm{~L}$ & -32 & -62 & 32 & 8.18 \\
\hline
\end{tabular}

The cluster-wise threshold (correct $p<.05)$ was determined by the voxel-wise threshold $(p<.001)$ and the cluster size $\left(25\right.$ contiguous voxels; $\left.200 \mathrm{~mm}^{3}\right)$. ${ }^{a}$ The activations of the dorsolateral prefrontal cortex and anterior insular cortex were not observed separately at this cluster-wise threshold. However, these activations were separable, and both were statistically significant at the conservative voxel-wise threshold $(p<.00001)$ and smaller minimum volume (five contiguous voxels; $40 \mathrm{~mm}^{3}$ ) that corresponded to corrected $p<.05$

The results of the parametric analysis (Table 7) showed that when a curiosity-inducing question or competence-enabling anagram was perceived to be relatively interesting, the left AIC was more activated $(p<.005$ with at least 52 contiguous voxels; corrected $p<.05)$. Together with the AIC, the left thalamus, right cerebellum, left DLPFC, left medial frontal gyrus, right inferior frontal gyrus, left postcentral gyrus, left precuneus, left fusiform gyrus, and bilateral occipital lobe also showed greater activations as the curiosity-inducing question or competence-enabling anagram was being perceived to be

Table 7 Results of the parametric analysis during the performance of intrinsically motivating tasks, using the degree of perceived intrinsic interest as a modulating parameter

\begin{tabular}{|c|c|c|c|c|c|c|c|}
\hline \multirow[t]{2}{*}{ Brain Region } & \multirow[t]{2}{*}{ BA } & \multirow[t]{2}{*}{ Volume } & \multirow[t]{2}{*}{ Side } & \multicolumn{3}{|c|}{ MNI Coordinates } & \multirow{2}{*}{$\begin{array}{l}\text { Maximum } \\
t \text { Value }\end{array}$} \\
\hline & & & & $x$ & $y$ & $z$ & \\
\hline Anterior insular cortex & 13 & 568 & $\mathrm{~L}$ & -30 & 24 & 0 & 4.52 \\
\hline Thalamus & & 904 & $\mathrm{~L}$ & -2 & -16 & 2 & 4.71 \\
\hline \multirow[t]{2}{*}{ Cerebellum } & & 880 & $\mathrm{R}$ & 36 & -66 & -26 & 3.90 \\
\hline & & 536 & $\mathrm{R}$ & 10 & -76 & -32 & 4.67 \\
\hline Dorsolateral prefrontal cortex & 46 & 14,296 & $\mathrm{~L}$ & -48 & 32 & 18 & 5.56 \\
\hline Medial frontal gyrus & 6 & 5,888 & $\mathrm{~L}$ & -4 & 4 & 60 & 5.07 \\
\hline Inferior frontal gyrus & 47 & 2,520 & $\mathrm{R}$ & 42 & 32 & -6 & 4.21 \\
\hline Postcentral gyrus & 2 & 552 & $\mathrm{~L}$ & -42 & -28 & 48 & 5.09 \\
\hline Precuneus & 7 & 2,992 & $\mathrm{~L}$ & -22 & -70 & 36 & 4.17 \\
\hline Fusiform gyrus & 37 & 2,896 & $\mathrm{~L}$ & -52 & -48 & -16 & 5.01 \\
\hline \multirow[t]{4}{*}{ Occipital lobe } & 18 & 3,936 & $\mathrm{R}$ & 24 & -88 & -6 & 4.99 \\
\hline & 18 & 568 & $\mathrm{~L}$ & -16 & -94 & -12 & 3.96 \\
\hline & 19 & 1,520 & $\mathrm{~L}$ & -28 & -78 & -22 & 4.48 \\
\hline & 17 & 552 & $\mathrm{~L}$ & -18 & -84 & 0 & 4.38 \\
\hline
\end{tabular}

The cluster-wise threshold (correct $p<.05)$ was determined by the voxel-wise threshold $(p<.005)$ and the cluster size $(n \geq 52$, a minimum volume of 416 $\mathrm{mm}^{3}$ ) 
more interesting $(p<.005$ with at least 52 contiguous voxels; corrected $p<.05$ ). No brain region showed significantly greater deactivation as the curiosity-inducing question or competence-enabling anagram was being perceived to be more interesting.

PPI analyses revealed that the right striatum (peak coordinates of the seed brain region: $10,-2,-8$; volume: $656 \mathrm{~mm}^{3}$ ) showed greater positive interactions with the left AIC (peak MNI coordinate: $-36,10,0$; maximum $t$ value $=4.51$; volume: $480 \mathrm{~mm}^{3}$; Fig. 4) and the right fusiform gyrus (peak MNI coordinate: $38,-50,-14$; maximum $t$ value $=4.52$; volume: $432 \mathrm{~mm}^{3}$ ) during the performance of intrinsically motivating tasks than during the performance of non-intrinsicallymotivating tasks $(p<.005$ with at least 52 contiguous voxels; corrected $p<.05$ ). No brain region showed significantly greater negative correlation with the right striatum during the performance of intrinsically motivating tasks than during the performance of non-intrinsically-motivating tasks.

In the case of the left striatum (peak coordinates of the seed brain region: $-20,2$, 8; volume: $608 \mathrm{~mm}^{3}$ ), no brain region showed significantly greater positive correlation with this brain region during the performance of intrinsically motivating tasks than during the performance of non-intrinsically-motivating tasks. In contrast, the left striatum showed greater negative correlations with the left inferior temporal gyrus (peak MNI coordinate: $-56,-34,-4$; maximum $t$ value $=4.29$; volume: $1,000 \mathrm{~mm}^{3}$ ) and right postcentral gyrus (peak MNI coordinate: $70,-18,24$; maximum $t$ value $=4.49$; volume: $520 \mathrm{~mm}^{3}$ ) during the performance of intrinsically motivating tasks than during the performance of non-intrinsically-motivating tasks ( $p$ $<.005$ with at least 52 contiguous voxels; corrected $p<.05$ ).

\section{Discussion}

We sought to identify the neural substrates of intrinsic motivation during actual task performance. To do so, we operationally defined intrinsic motivation in two ways that allowed us to fully represent the conceptual definition of intrinsic motivation - namely, answering curiosity-inducing (vs. noncuriosity-inducing) questions and solving competenceenabling (vs. non-competence-enabling) anagrams. When we examined participants' neural activity under both of these conditions, we found that the intrinsic-motivation neural system involved (1) AIC activity (see Fig. 3A), (2) striatum activity (see Fig. 3B), and (3) AIC-striatum interactions (see Fig. 4).

The AIC activation during intrinsically motivating activities was consistent with previous findings showing that the AIC is recruited when participants imagined that they were engaging in intrinsically motivating tasks or activities (Lee \& Reeve, 2013; Lee et al., 2012). Therefore, AIC activity seems to be an important part of the intrinsic-motivation system not only when people imagine experiencing, but also when they actually experience, intrinsic motivation. The AIC is known to be associated with the processes of "subjective feelings from the body" (Craig, 2009; Damasio, 1999; Damasio \& Carvalho, 2013). Specifically, it represents internal bodily needs (e.g., hunger, drug craving), monitors bodily states related to these needs (e.g., satiation, deprivation), and integrates the bodily information into subjective feelings (Goldstein et al., 2009; Naqvi \& Bechara, 2009; Naqvi, Rudrauf, Damasio, \& Bechara, 2007). We therefore conclude that AIC activity from feelings of intrinsic satisfaction is a key source of intrinsic motivation, a conclusion further supported by the positive correlation between participants' self-reported ongoing (trial-by-trial) task interest and the extent of AIC activity when participants performed intrinsically motivating tasks.

Striatum activity was also recruited when participants engaged in tasks designed to enhance intrinsic motivation during task performance. The striatum is known to play a crucial role in reward processing (Delgado, 2007; Haber \& Knutson, 2010; O'Doherty, 2004). That is, it receives and integrates reward-related information from cortical and subcortical regions and produces approach behaviors based on this reward-related information. In this regard, contemporary neuroscience suggests that striatum activity is a central part of

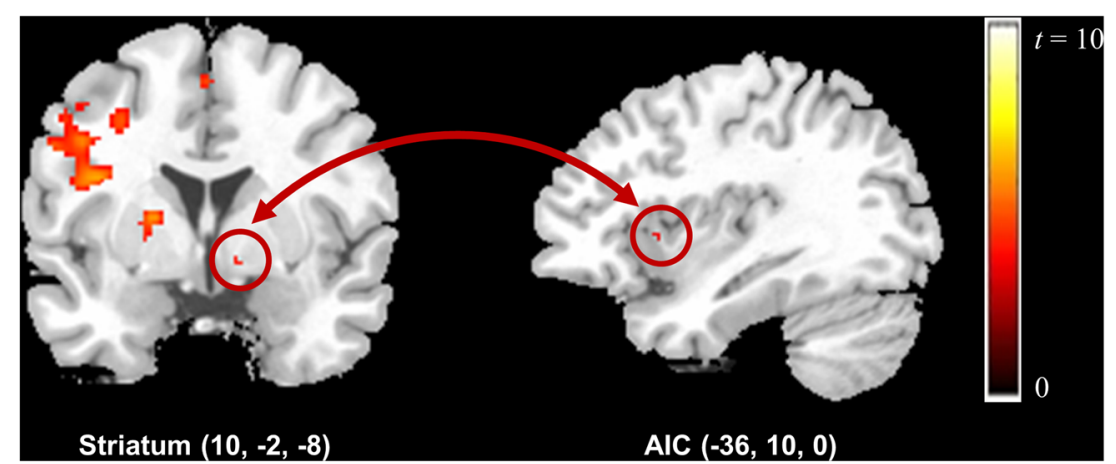

Fig. 4 Right striatum showed greater positive interaction with the left AIC during the performance of intrinsically motivating tasks than during the performance of non-intrinsically-motivating tasks $(p<.005$ with at least 52 contiguous voxels; corrected $p<.05)$ 
extrinsically generated motivation (Berridge, 2004; Cardinal, Parkinson, Hall, \& Everitt, 2002; Liljeholm \& O'Doherty, 2012). Recent neuroscience studies have reported, however, that the striatum is activated not only when participants experience extrinsic motivation during task performance, but also when they experience intrinsic motivation during task performance (e.g., resolving curiosity, feeling competent; Gruber et al., 2014; Kang et al., 2009; Murayama et al., 2010). We therefore conclude that striatum activity during tasks rated as suspenseful, challenging, satisfying, and interesting (see Fig. 1) generates "intrinsic" reward.

We observed AIC-striatum interactions during intrinsically motivating activities. That is, AIC and striatum activities positively interacted more during the performance of intrinsically, relative to non-intrinsically, motivating tasks. This finding is consistent with previous neuroscientific findings showing functional connectivity between the AIC and striatum (Cho et al., 2013; Postuma \& Dagher, 2006). This functional connectivity pattern is consistent with well-established anatomical connections between the AIC and striatum (Chikama et al., 1997; Singer, Critchley, \& Preuschoff, 2009). Some researchers have viewed this connectivity as the integration of bodily information and reward-related information during the processes of subjective feelings (Craig, 2009). In contrast, others have viewed this connectivity as the process that some subjective feelings lead to approach behaviors (Cho et al., 2013; Damasio \& Carvalho, 2013; Naqvi \& Bechara, 2009). Despite these somewhat different views, the functional connectivity result observed in the present study suggests that the interaction between AIC and striatum activities shows that the intrinsic-motivation system involves feelings of inherent satisfaction, intrinsic reward processing, and an exchange of information between these feelings of interest and intrinsic reward processing. As such, the findings from the PPI analysis show that not only is the AIC involved in the intrinsic-motivation system (Lee \& Reeve, 2013; Lee et al., 2012) and not only is the striatum involved in the intrinsic-motivation system (Gruber et al., 2014; Kang et al., 2009; Murayama et al., 2010), but these two brain areas work together interactively (rather than independently) in the intrinsic-motivation system.

These findings help identify what intrinsic motivation is and is not. As we introduced in the article's opening paragraph, intrinsic motivation is, theoretically and conceptually, the inherent tendency to seek out novelty and challenge, to explore and investigate, and to stretch and extend one's capacities. In the present study, we offered participants activities that were (1) suspenseful, curiosity-satisfying, and interesting and (2) optimally challenging, competence satisfying, and interesting (Figs. 1A and B). These same intrinsic-motivationgenerating stimuli were also more difficult to solve than were their non-curiosity-inducing and non-competence-enabling counterparts. Task difficulty is not a defining characteristic of an intrinsically motivating activity; rather, it is inherently intertwined with learning new information and mastering optimal challenges, which are defining characteristics of an intrinsically motivating activity. This is why we elected to statistically control for task difficulty as a potentially confounding stimulus characteristic. Our analysis that controlled for task difficulty confirmed that novelty and challenge were defining characteristics, whereas task difficulty was incidental (Table 6). That said, other stimulus properties beyond task difficulty might also be inherently intertwined with the piquing of curiosity (e.g., how surprising or arousing the stimulus is) and with the offering of optimal challenge (e.g., how information-rich or feedback-generating the stimulus is).

We encourage future research to investigate these possible stimulus confounds (in the same way we investigated the incidental status of task difficulty in the present study), but our position on what intrinsic motivation is - based on the extensive literature in psychology (Ryan \& Deci, 2017) — is the following: Encountering environmental novelty, having an opportunity to discover new information, feeling suspense over what might come next, anticipating satisfaction from attaining new information, actually assimilating that new information, and solving a mystery collectively activate intrinsic motivation (via curiosity anticipation and satisfaction), just as encountering optimal challenge, having an opportunity to stretch and expand one's skills, feeling enjoyment from being challenged, anticipating satisfaction from a job well done, actually experiencing mastery and effectiveness from making progress, and developing greater capacity also collectively activate intrinsic motivation (via competence anticipation and satisfaction; Abuhamdeh \& Csikszentmihalyi, 2012; Abuhamdeh et al., 2015; Deci \& Ryan, 1985; Loewenstein, 1994; White, 1959). This conceptualization of intrinsic motivation as the pursuit and satisfaction of intrinsic rewards is consistent not only with the psychological literature but also with the functional role of the AIC in the pursuit, monitoring, and satisfaction of intrinsic rewards (Craig, 2009; Naqvi \& Bechara, 2009), such as when people assimilate new information (seeking and satisfying curiosity) and when they master optimal challenges (seeking and satisfying competence).

Greater activation of frontal areas (e.g., DLPFC, medial frontal gyrus) was also observed when participants performed intrinsically motivating tasks than when they performed nonintrinsically-motivating tasks. Considering these frontal areas are well-known regions for higher-order cognitive processes (e.g., cognitive control, performance monitoring) (Carter et al., 1998; Miller \& Cohen, 2001), the experiences of intrinsic motivation may play a role in mobilizing cognitive processes during task performance. This interpretation was supported by the results of the supplementary subtraction analysis, that increased activations of these frontal areas during the performance of intrinsically motivating tasks were also observed even after controlling for the effects of the difficulty 
of each question or anagram (see Table 6). This interpretation was also supported by the results of the parametric analyses, that these frontal region activations were positively correlated with participants' interest during the performance of intrinsically motivating tasks, whereas they were not significantly correlated with the difficulty of each curiosity-inducing question or competence-enabling anagram. This means that people generated more mental effort during task performance not as the question or anagram became more difficult, but instead as they experienced greater task interest (intrinsic motivation).

This study has possible limitations that both constrain the interpretation of the present findings and invite future research to determine how much of a concern the limitations might be. The first limitation was that our experimental manipulations to create curiosity and competence satisfactions involved not only core intrinsically motivated processes but also the cognitive effort required to achieve these satisfactions (answer the questions, solve the anagrams). That is, our experimental conditions stimulated both intrinsic motivation and mental effort, whereas our control conditions stimulated neither intrinsic motivation nor mental effort. This was true in our investigation, and it was also true in the previous investigations of intrinsic motivation involving curiosity-inducing questions (Gruber et al., 2014; Kang et al., 2009) and competenceenabling challenges (Murayama et al., 2010). Therefore, it is important to know whether intrinsic motivation manipulated by other experimental paradigms might also recruit the neural circuit observed in this study. One way to do this would be to have participants all engage in exactly the same task (to control for cognitive effort requirements) but for two different reasons, such as intrinsic-motivation-facilitating reasons (e.g., "do it only for personal interest") versus intrinsicmotivation-suppressing but extrinsic-motivation-facilitating reasons (e.g., "do it only for monetary compensation"). We suggest this research question for future investigations.

A second limitation was that our experimental design (Fig. 2) left us unable to separate the neural activations when participants experienced presolution suspense and challenge versus when participants experienced postsolution curiosity and competence satisfaction. In our research design, the presolution and postsolution phases were closely linked because participants knew that satisfying solutions to the questions and anagrams would be forthcoming in seconds. Given this, we elected to study intrinsic motivation as a unitary experience and to analyze participants' neural activity during the last few seconds of each trial, which represented the maximum point of participants' curiosity and competence satisfaction. But a different research design is possible. With both curiosity and competence, an initial presolution suspense and challenge phase is then followed by a postsolution curiosity and competence satisfaction phase. These two phases within an episode of intrinsic motivation could be investigated separately, by introducing a jittered interstimulus interval between the presolution and postsolution phases, for instance. Such a research design could address the question as to whether or not curiosity and competence might be understood as two distinct motivational events that feature important early-phase and late-phase neural differences.

A third limitation to acknowledge would be our lack of an objective behavioral measure of intrinsic motivation. It is common in laboratory investigations of intrinsic motivation to assess intrinsic motivation through both a self-report interest rating and a "free-choice behavioral measure of intrinsic motivation," which is the amount of time that a participant plays with the experimental task during a postexperimental free-choice period (Deci et al., 1999). Our particular experimental paradigm did not lend itself well to the inclusion of such a behavioral measure, but we acknowledge that future studies could offer participants postscanner questions and anagrams to confirm that the curiosity-inducing questions and competence-enabling anagrams were intrinsically motivating, whereas the non-curiosity-inducing questions and noncompetence-enabling anagrams were not.

Finally, we found functional connectivity between the right striatum and the left AIC, whereas we did not find functional interactions of the left striatum with the AIC. We did not formulate an a priori hypothesis regarding this, because previous findings could not suggest consistent hemispheric differences in AIC-striatum interactions related to intrinsic motivation. However, these hemispheric differences seem to be an interesting research question for future investigations.

Despite these possible limitations, we found that AIC activity, striatum activity, and their functional interactions are key components of the intrinsic-motivation neural system. This means that AIC activity and striatum activity are both important in the intrinsic-motivation neural system, and it also means that the interactive roles of these two brain regions are also critical. So the intrinsic-motivation neural system does reflect reward processing (associated with striatum activity), but it is also clearly distinct from the extensively investigated striatum reward system, in that AIC and AIC-striatum interactions are additional key inputs.

Author note This work was supported by the Ministry of Education of the Republic of Korea and the National Research Foundation of Korea (NRF-2015S1A5B6036594).

\section{References}

Abuhamdeh, S., \& Csikszentmihalyi, M. (2012). The importance of challenge for the enjoyment of intrinsically motivated, goal-directed activities. Personality and Social Psychology Bulletin, 38, 317-330.

Abuhamdeh, S., Csikszentmihalyi, M., \& Jalal, B. (2015). Enjoying the possibility of defeat: Outcome uncertainty, suspense, and intrinsic motivation. Motivation and Emotion, 39, 1-10. 
Berridge, K. C. (2004). Motivation concepts in behavioral neuroscience. Physiology \& Behavior, 81, 179-209. doi:10.1016/j.physbeh.2004. 02.004

Cardinal, R. N., Parkinson, J. A., Hall, J., \& Everitt, B. J. (2002). Emotion and motivation: The role of the amygdala, ventral striatum, and prefrontal cortex. Neuroscience \& Biobehavioral Reviews, 26, 321-352.

Carter, C. S., Braver, T. S., Barch, D. M., Botvinick, M. M., Noll, D., \& Cohen, J. D. (1998). Anterior cingulate cortex, error detection, and the on-line monitoring of performance. Science, 280, 747-749.

Cerasoli, C. P., Nicklin, J. M., \& Ford, M. T. (2014). Intrinsic motivation and extrinsic incentives jointly predict performance: A 40-year meta-analysis. Psychological Bulletin, 140, 980-1008.

Chikama, M., McFarland, N. R., Amaral, D. G., \& Haber, S. N. (1997). Insular cortical projections to functional regions of the striatum correlate with cortical cytoarchitectonic organization in the primate. Journal of Neuroscience, 15, 9686-9705.

Cho, Y. T., Fromm, S., Guyer, A. E., Detloff, A., Pine, D. S., Fudge, J. L., \& Ernst, M. (2013). Nucleus accumbens, thalamus and insula connectivity during incentive anticipation in typical adults and adolescents. NeuroImage, 66, 508-521.

Clifford, M. M. (1990). Students need challenge, not easy success. Educational Leadership, 48, 22-26.

Cox, R. W. (1996). AFNI: Software for analysis and visualization of functional magnetic resonance neuroimages. Computers and Biomedical Research, 29, 162-173.

Craig, A. D. (2009). How do you feel-now? The anterior insula and human awareness. Nature Reviews Neuroscience, 10, 59-70. doi:10. $1038 / \mathrm{nrn} 2555$

Damasio, A. R. (1999). The feeling of what happens: Body and emotion in the making of consciousness. New York: Harcourt.

Damasio, A., \& Carvalho, G. B. (2013). The nature of feelings: Evolutionary and neurobiological origins. Nature Reviews Neuroscience, 14, 143-152.

Decety, J. (1996). The neurophysiological basis of motor imagery. Behavioral Brain Research, 77, 45-52.

Deci, E. L. (1992). The relation of interest to the motivation of behavior: A self-determination theory perspective. In K. A. Renninger, S. Hidi, \& A. Krapp (Eds.), The role of interest in learning and development (pp. 43-60). Hillsdale: Erlbaum.

Deci, E. L., Koestner, R., \& Ryan, R. M. (1999). A meta-analytic review of experiments examining the effects of extrinsic rewards on intrinsic motivation. Psychological Bulletin, 125, 627-668. doi:10.1037/ 0033-2909.125.6.627

Deci, E. L., \& Ryan, R. M. (1985). Intrinsic motivation and selfdetermination in human behavior. New York: Plenum.

Delgado, M. R. (2007). Reward-related responses in the human striatum. In B. W. Balleine, K. Doya, J. O’Doherty, \& M. Sakagami (Eds.), Reward and decision making in corticobasal ganglia networks (Annals of the New York Academy of Sciences (Vol. 1104, pp. 7088). Malden: Blackwell.

Esterman, M., Tamber-Rosenau, B. J., Chiu, Y. C., \& Yantis, S. (2010). Avoiding nonindependence in fMRI data analysis: Leave one subject out. NeuroImage, 50, 572-576.

Faul, F., Erdfelder, E., Lang, A.-G., \& Buchner, A. (2007). G*Power 3: A flexible statistical power analysis program for the social, behavioral, and biomedical sciences. Behavior Research Methods, 39, 175-191. doi:10.3758/BF03193146

Forman, S. D., Cohen, J. D., Fitzgerald, M., Eddy, W. F., Mintun, M. A., \& Noll, D. C. (1995). Improved assessment of significant activation in functional magnetic resonance imaging (fMRI): Use of a clustersize threshold. Magnetic Resonance in Medicine, 33, 636-647.

Friston, K. J., Buechel, C., Fink, G. R., Morris, J., Rolls, E., \& Dolan, R. J. (1997). Psychophysiological and modulatory interactions in neuroimaging. NeuroImage, 6, 218-229.
Goldstein, R. Z., Craig, A. D., Bechara, A., Garavan, H., Childress, A. R., Paulus, M. P., \& Volkow, N. D. (2009). The neurocircuitry of impaired insight in drug addiction. Trends in Cognitive Sciences, 13, 372-380.

Gruber, M. J., Gelman, B. D., \& Ranganath, C. (2014). States of curiosity modulate hippocampus-dependent learning via the dopamine circuit. Neuron, 84, 486-496.

Haber, S. N., \& Knutson, B. (2010). The reward circuit: Linking primate anatomy and human imaging. Neuropsychopharmacology, 35, 4 26. doi:10.1038/npp.2009.129

Jang, H., Kim, E. J., \& Reeve, J. (2016). Why students become more engaged or more disengaged during the semester: A selfdetermination theory dual-process model. Learning and Instruction, 43, 27-38.

Jeannerod, M. (2001). Neural simulation of action: A unifying mechanism for motor cognition. NeuroImage, 14, S103-S109.

Kang, M. J., Hsu, M., Krajbich, I. M., Loewenstein, G., McClure, S. M., Wang, J. T., ... \& Camerer, C. F. (2009). The wick in the candle of learning: epistemic curiosity activates reward circuitry and enhances memory. Psychological Science, 20, 963-973. doi:10.1111/j.14679280.2009.02402.x

Kawabata, M., \& Mallett, C. J. (2011). Flow experience in physical activity: Examination of the internal structure of flow from a processrelated perspective. Motivation and Emotion, 35, 393-402.

Krapp, A. (2005). Basic needs and the development of interest and intrinsic motivational orientations. Learning and Instruction, 15, 381395.

Kriegeskorte, N., Simmons, W. K., Bellgowan, P. S. F., \& Baker, C. I. (2009). Circular analysis in systems neuroscience: The dangers of double dipping. Nature Reviews Neuroscience, 12, 535-540.

Lee, W., \& Reeve, J. (2013). Self-determined, but not non-self-determined, motivation predicts activations in the anterior insular cortex: An fMRI study of personal agency. Social Cognitive and Affective Neuroscience, 8, 538-545.

Lee, W., Reeve, J., Xue, Y., \& Xiong, J. (2012). Neural differences between intrinsic reasons for doing versus extrinsic reasons for doing: An fMRI study. Neuroscience Research, 73, 68-72.

Lieberman, M. D., \& Cunningham, W. A. (2009). Type I and Type II error concerns in $\mathrm{MMRI}$ research: Rebalancing the scale. Social Cognitive and Affective Neuroscience, 4, 423-428. doi:10.1093/scan/nsp052

Liljeholm, M., \& O’Doherty, J. P. (2012). Contributions of the striatum to learning, motivation, and performance: An associative account. Trends in Cognitive Sciences, 16, 467-475.

Loewenstein, G. (1994). The psychology of curiosity: A review and reinterpretation. Psychological Bulletin, 116, 75-98.

Miller, E. K., \& Cohen, J. D. (2001). An integrative theory of prefrontal cortex function. Annual Review of Neuroscience, 24, 167-202. doi: 10.1146/annurev.neuro.24.1.167

Murayama, K., Matsumoto, M., Izuma, K., \& Matsumoto, K. (2010). Neural basis of the undermining effect of monetary reward on intrinsic motivation. Proceedings of the National Academy of the Sciences, 107, 20911-20916. doi:10.1073/pnas.1013305107

Naqvi, N. H., \& Bechara, A. (2009). The hidden island of addiction: The insula. Trends in Neurosciences, 32, 56-67. doi:10.1016/j.tins.2008. 09.009

Naqvi, N. H., Rudrauf, D., Damasio, H., \& Bechara, A. (2007). Damage to the insula disrupts addiction to cigarette smoking. Science, 315, 531-534. doi:10.1126/science.1135926

O'Doherty, J. P. (2004). Reward representations and reward-related learning in the human brain: Insights from neuroimaging. Current Opinion in Neurobiology, 14, 769-776.

Postuma, R. B., \& Dagher, A. (2006). Basal ganglia functional connectivity based on a meta-analysis of 126 positron emission tomography and functional magnetic resonance imaging publications. Cerebral Cortex, 16, 1508-1521. 
Reeve, J. (1989). The interest-enjoyment distinction in intrinsic motivation. Motivation and Emotion, 13, 83-103.

Reeve, J., \& Lee, W. (2012). Neuroscience and human motivation. In R. M. Ryan (Ed.), Oxford handbook of human motivation (pp. 365380). New York: Oxford University Press.

Reeve, J., \& Nix, G. (1997). Expressing intrinsic motivation through acts of exploration and facial displays of interest. Motivation and Emotion, 21, 237-250.

Ryan, R. M., \& Deci, E. L. (2000). Self-determination theory and the facilitation of intrinsic motivation, social development, and wellbeing. American Psychologist, 55, 68-78. doi:10.1037/0003066X.55.1.68

Ryan, R. M., \& Deci, E. L. (2017). Self-determination theory: Basic psychological needs in motivation, development, and wellness. New York: Guilford Press.
Silvia, P. J. (2008). Interest: The curious emotion. Current Directions in Psychological Science, 17, 57-60.

Singer, T., Critchley, H. D., \& Preuschoff, K. (2009). A common role of insula in feelings, empathy, and uncertainty. Trends in Cognitive Sciences, 13, 334-340.

Vansteenkiste, M., \& Deci, E. L. (2003). Competitively contingent rewards and intrinsic motivation: Can losers remain motivated? Motivation and Emotion, 27, 273-299.

Vansteenkiste, M., Simons, J., Lens, W., Sheldon, K. M., \& Deci, E. L. (2004). Motivating learning, performance, and persistence: The synergistic role of intrinsic goals and autonomy support. Journal of Personality and Social Psychology, 87, 246-260.

White, R. W. (1959). Motivation reconsidered: The concept of competence. Psychological Review, 66, 297-333. 